\title{
A Social Robotic Obesity Management and Awareness System for Children in Saudi Arabia
}

\author{
https://doi.org/10.3991/ijoe.v14i09.9015 \\ Mohammed Alotaibi \\ University of Tabuk, Saudi Arabia \\ malotaibilut.edu.sa
}

\begin{abstract}
The prevalence of obesity and overweight among children has increased significantly during last two decades in Saudi Arabia with overwhelming consequences to public health. Most recommended approaches to reduce obesity have paying attention on healthier diet and physical Activity (PA). The recent research shows that the use of social robots can contribute in fulfilling all the needs to encourage children to improve their skills in selfmanagement. As children need to be surprised and feel a sense of enjoyment when involving in any activity where they can spend time, effort and be dedicated to. Hereby, this paper presents an innovation social Robotic system to offer a set of activities to help obese children in improving their capabilities to manage their selves properly and increase their obesity knowledge. The proposed system presented in this paper will be tested and evaluated extensively in a random controlled trial in Saudi Arabia.
\end{abstract}

Keywords — obesity, m-health; asthma Management; telemedicine; e-health; Kingdom of Saudi Arabia

\section{Introduction}

Obesity has been become a one of the most common chronic disease during the last two decades due to the big change in people lifestyle globally. Obesity is measured by using the body mass index value. Herby, the body mass index (BMI) is an indicator calculated from the mass (weight) and height of an individual. The BMI is defined as "the body mass divided by the square of the body height, and is universally expressed in units of $\mathrm{kg} / \mathrm{m} 2$, resulting from mass in kilograms and height in meters". [1].

According to world health organization [2] over 1.9 billion adults, 18 years and older, were overweight globally in 2016. Of these over 650 million were obese. Moreover, around 41 million of children whom under 5 years are obese or overweight worldwide. Nearly half of the obese or overweight children were lived in Asia [2]. Obesity Atlas $33.4 \%$ of female and $24.1 \%$ of male are overweight or obese in 2016 [3]. Furthermore, the prevalence of obese and overweight children whom between 4 and 8 years old in Saudi are around 19.2\%. [3] as shown in figure 1.

Moreover, many studies [4, 5, and 6] reported that there is a big relation between obesity and other chronic disease like diabetes and hypertension. Thereby, this means 
the obese children are in danger of getting diabetes in their early age. Modern lifestyle changes, less physical activity and Dietary habits are consider as the most important reasons behind the high prevalence of obesity and overwrite among children in Saudi [7]. This means Saudi government has to take hardstand to decrease the prevalence of obesity especially among children by focusing on the awareness as initial phase. In consequence, the use of modern technology such as internet of things, mobile health and social robotics might be excellence solutions to improve the society awareness and cost-effective solution.

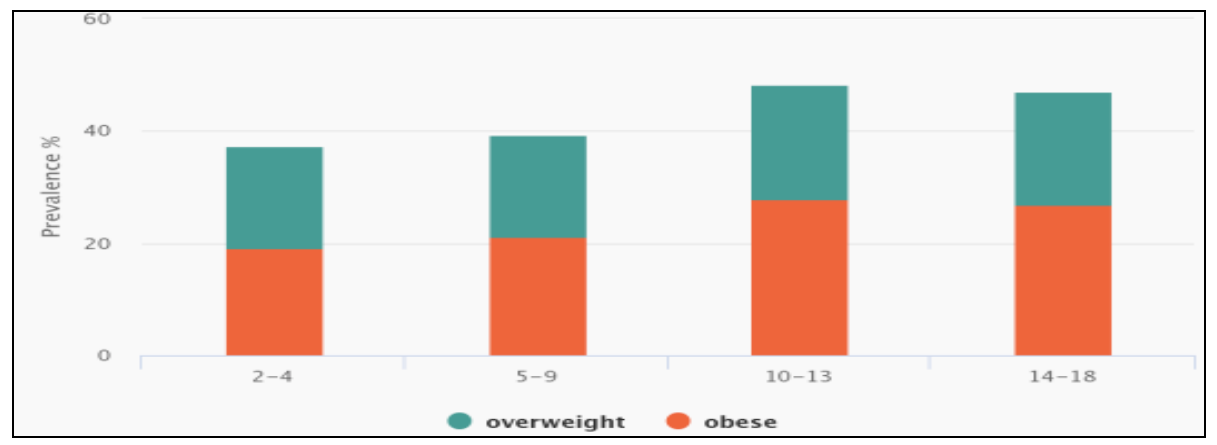

Fig. 1. .Prevalence of obese and overweight children between 4 and 8 in Saudi Arabia

The fast development of telecommunication and internet of things technology, smart mobile devices and Robotics technology has accelerated the design and implementation of healthcare services delivery for the management and awareness of various chronic diseases such obesity and diabetes. In addition, children requirements are different to adult as they need something surprise them, encourage them and attractive to. Hereby, the use of robotic technology can provide the children needs while the social robots can attractive them.

Some studies reported that the use of social robotics played main role in improving the chronic disease management like diabetes awareness. A systematic review study [8] focused on the role of using social robotic in improving diabetes management among children, six studies which focus on the use of social robotic in improving diabetes management, diabetes awareness or both diabetes management and awareness in different counties. The study concludes that the use of such technology has played an excellence role in improving diabetes awareness and management among children.

Moreover, a study [9] measured the Acceptability of using the humanoid robot as an assistant in their diabetes management process in the United Kingdom in 2016. 37 diabetic children aged between 6 to 16 years were participated in the study during their clinic visited. They stayed with the robot for a half an hour. The study outcomes showed that the overall children' acceptability is $86.7 \%$. Though, the level of acceptability vary based on the age group; children aged between 6 and 9 years had the highest acceptability level of $94.8 \%$, whereas the children aged groups between 10-12 and 13-16 years, had lower acceptability levels of $85.0 \%$ and $83.0 \%$, respectively. 
To date, there has not been a social Robot to enhance obesity management and awareness among children Saudi Arabia and other gulf countries. Innovative strategies to address the needs of obese and overweight children are urgently essential to deliver health services and medical education in attractive and cost-effective way. This project is trying to fill the research gab of using social Robotics in enhancing obesity management and awareness among children in Saudi Arabia.

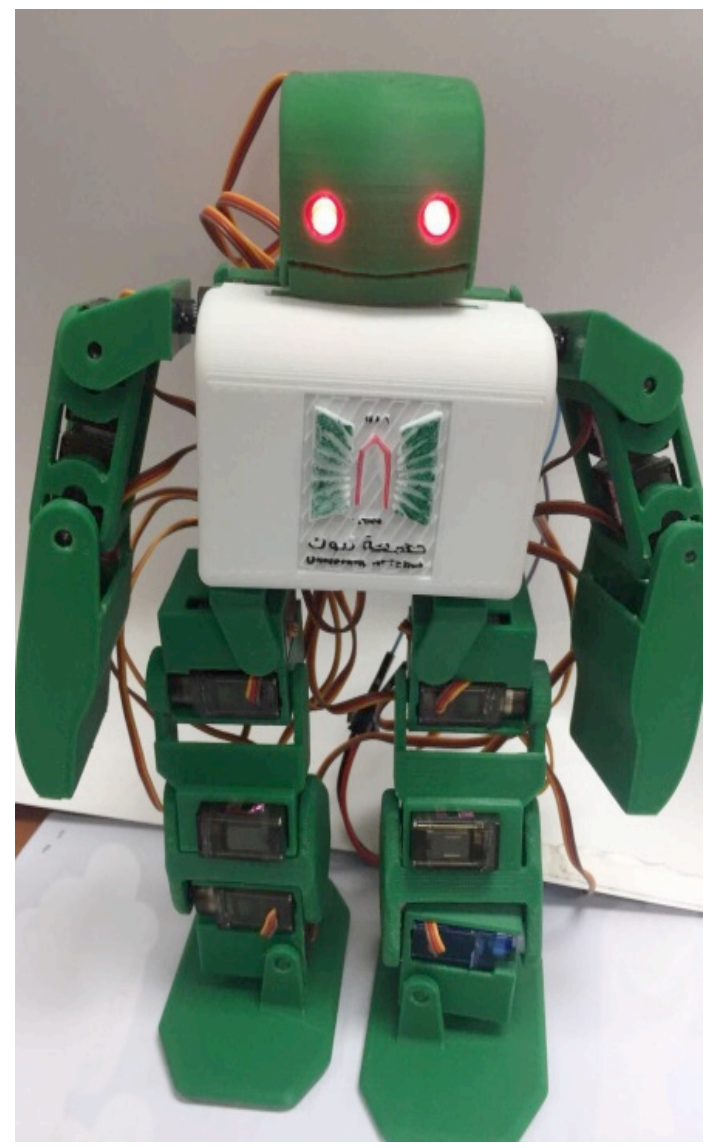

Fig. 2. Social Robot body

This paper presents the system architecture of a social Robot for enhancing obesity management among children in Saudi. The remainder of the paper is organized as follows: Section II describes the methodology, including the system overview and implementation, and Section III provides a discussion, conclusions and future work. 


\section{$2 \quad$ Method}

\subsection{The System's Clinical Requirements and Design Analysis.}

To implement and design the Social Robots system for enhancing the obesity management and awareness among children in Saudi, an extensive discussion was carried out with paediatric doctors from the faculty of Medicine at the University of Tabuk to locate the most important system functional requirements. They recommended the following system functional requirements:

1. The Social robots design should be suitable and attractive for children.

2. The system should encourage the overweight and obese children to reach a specific target of physical Activity weekly specially the walking activity. And also the target of weight reduction weekly.

3. The social Robot should include a quiz exam regarding the obesity awareness while the Social Robot could ask the child some questions and get answers.

4. The social robot should deliver information about obesity and other related chronic disease to the children. They recommended the information in manner of stories which is suitable for children.

5. The system should follow up the children movement during the day. As they mention if the child does a specific exercise during the day for an hour but he is staying at home all the day without any activity. This does not mean he is an active.

6 . The system should include a competition between children whom involve in system.

7. Social robot should deliver suitable emotion regarding weight weekly measurement.

8. The social robot should remind the child to take the weight measurement weekly.

9. Parent should involve in the process to follow and motivate their child and get clear picture of their child health progress.

\subsection{System overview}

The social robotics obesity management and awareness system targeted for children in Saudi Arabia is depicted in Fig. 3 below. It consists of two components:

Social robotics patient/healthcare provider component. This component includes the mobile and robot end of the system developed using smart phone platform and robotic technology tools. It covers most of the interaction units of the system. Thereby, it includes the following stations:

Obese or overweight children and their parents unit: The unit typically includes (a) a smart mobile phone, (b) a wrist bond sensor and (c) the social robot. In this unit, the obese/overweight children are empowered with a smart mobile application that includes a data management environment that delivers a personal medical profile, a record of daily activity specially the number of steps and daily of consumed calories. And also the weekly weight measurements in the form of tables and charts. Moreover, researcher developed a wrist bond to calculate the number of walked steps during the 


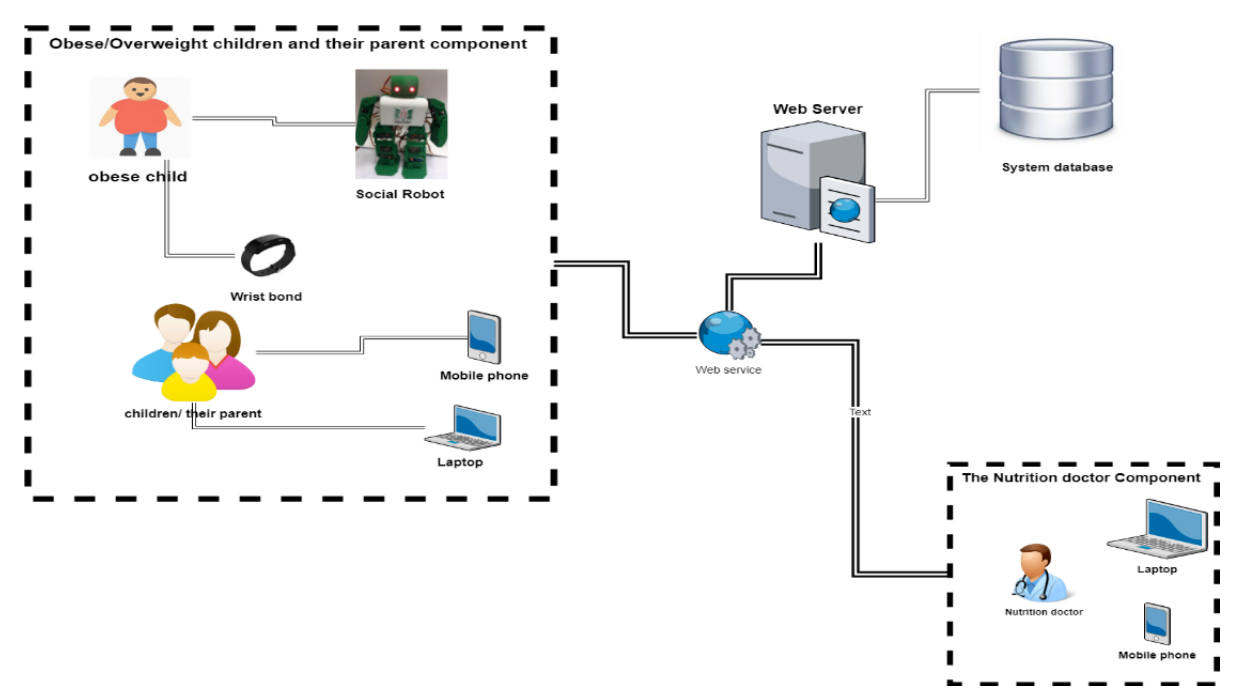

Fig. 3. system design

day. This technology is provided by the mobile accelerometer and also delivered through smart watches, but based on Saudi culture most of families do not prefer to have their child mobile phone and also the cost of smart phones in Saudi is considered so expensive. Moreover, the price of existing smart watches watch is so expensive which worth around 2400 Saudi riyal while the developed wrist bond cost does not exceed 60 Saudi riyals, more details about it is explained in the implementation section. In addition, the parents are involve in the system and can create account in the system to follow and involve in their child management and progress.

Social Robot: The implemented social robotics is connected with the whole system. It will be in the middle between the specialist nutrition and obese/overweight child and their parents. A quiz exam game is installed on the system while the social robot will ask the child a question with two option answers either Yes or No. when the child answered correctly the social robot emotion will appreciate him like smiling for example. Moreover, the social robot will receive the child daily count steps from the wrist bond that is wear by child. The social robots' technology will be discussed in the implementation section below.

Specialist diabetic nurse unit: This unit includes a user friendly mobile application to the nurses or clinicians involved in the diagnosis of obesity. It presents children obesity data management system which enables the clinician to communicate and follow up their obese/overweight children remotely. The nutrition can send recommendation to their patients remotely. The nutrition can also set up a target of daily/weekly activity specially the target of walked steps and also can set up a weekly target of weight reduction.

The admin unit: The admin unit is responsible for the system's repair and maintenance. It monitors the technical aspects of the system and is also responsible for maintaining the backup of the data collected via the system. 
Children obesity/overweight management component. This unit contains the back end operations of the whole system which mainly engages all process of collecting information or data and storing them in the database. Thereby, it can be used by various other modules such as the obese/overweight children and their parent front end and clinician/nutrition front unit, etc. This component includes Database module which stores information related to individual obese/overweight child records of measurements related to the laboratory examination results such as other chronic disease if exist. This unit also establishes communication with the information system of the hospital where the system deployed.

\section{System implementation and discussion}

As mentioned above, the whole system consists of main components: Social robotic system component, step counts component, and web-server and mobile platform component. Each component is discussed below.

\subsection{Mobile application and web portal:}

The mobile application and web portal are developed php programming language, MySQL database and SWIF for iOS devices and android studio for android. Developers insure that the system is carrying out all the functional requirements recommended by the medical staff as mentioned above. Some snapscreens of the mobile app are shown in Figures 4, 5, and 6.

Most of the system functions are carried out through the mobile application such as the weight record, clinical profile and the data share with doctor while the doctor can access the patient profile from the mobile application as shown in figure 6 .

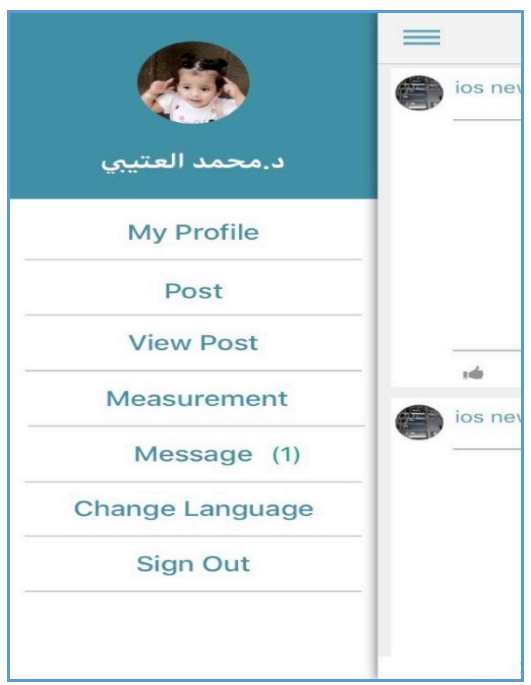

Fig. 4. The Mobile app control panel 


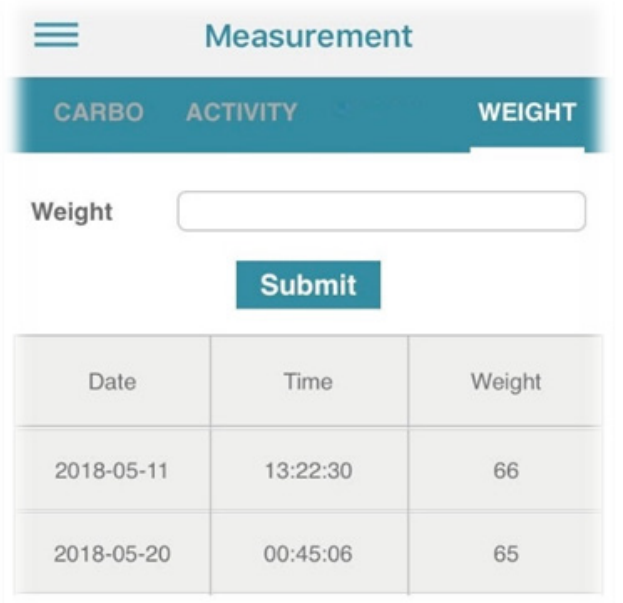

Fig. 5. The child weight logbook

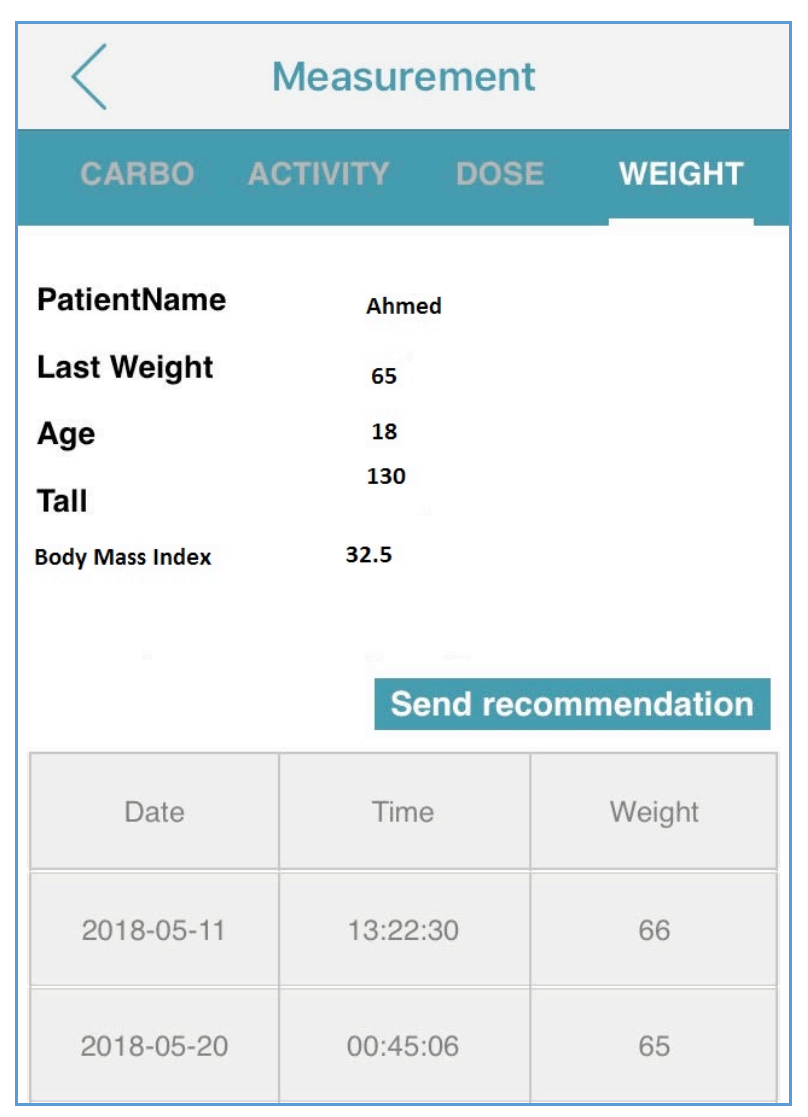

Fig. 6. Patient Profile at the Doctor end. 


\subsection{Social Robotic technology}

The robot's body is depicted in figure 2 above. The Robot body is designed in green color to be suitable with Saudi culture where the Saudi national flag is green. The university's logo is also included in the design as well. It has been printed using $3 \mathrm{D}$ printer at the Lab of robotic club at the faculty of computing and Information Technology, University of Tabuk. Figure 6 presents the social robot's architecture, where it contains of three layers: sensors layer, computing layer, communication layer, all are connected to power supply. Raspberry Pi 3 has been used as the brain of the robotic system and developed to offer five functionalities as depicted in Figure 6.

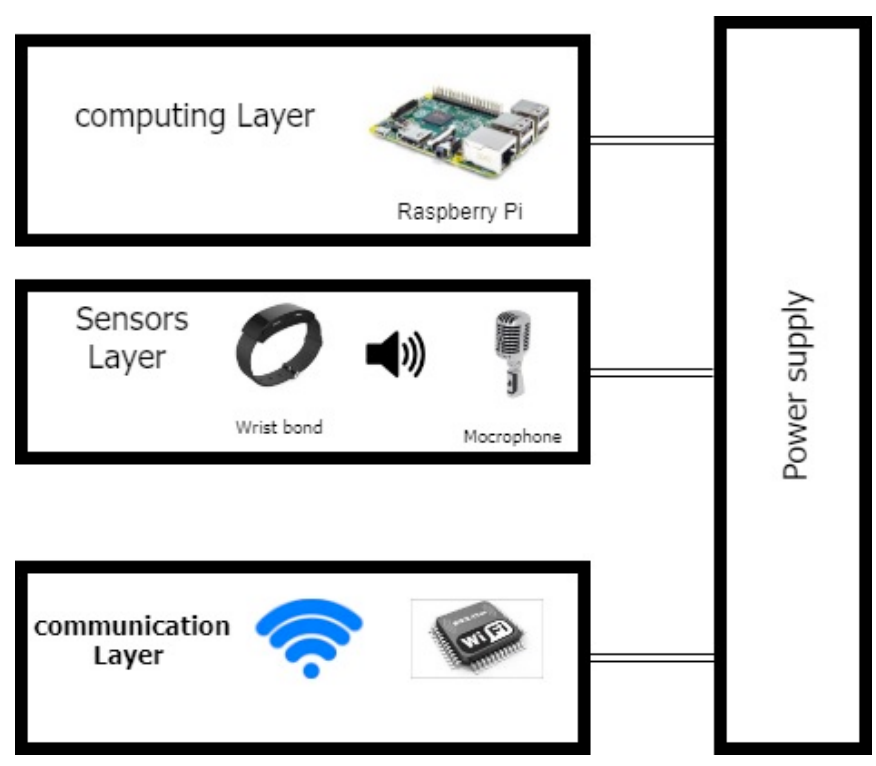

Fig. 7. Robot's architecture

Next, we describe each component and its main functionalities. The xx functionalities are illustrated as follows:

1. Obesity/overweight education: The robot will include some stories for children to improve their knowledge about obesity and healthy life. The child can ask the robot to tell him/her a stories and then the Robot will does. The social robot is smart enough to not repeat any story delivered before until all recorded stories completed.

2. Reminder system: Count of steps, number of consumed calories and body weight data must be collected regularly (for instance: daily or weekly), therefore the number of count steps will be transmitted automatically from the wrist bond to the robot through WIFI technology. If the child forgets to any of mentioned data above, the robot will remind him/her and parent will be reminded by the mobile app in manner of push notification message. 
3. Web-based system: The collected data from the robotic system is required to be available to the nutrition doctor anywhere and anytime. Therefore, the collected data will be transferred to a web-server.

4. Movement: The robotic system interacts with the child in an attractive manner, in order to make the system more effectual. Therefore, the robotic body consists of 10 servo motors. It can move and dance easily.

5. Quiz game: The social Robot can play a quiz game with child. The child from the mobile application can communicate with the Robot to play a quiz exam. A list of multiples choices questions will be available to child from the mobile screen, when a child selected and answer, the robot will give him a motivated emotion(dancing) if his/her answer is correct and will not dance if the answer if incorrect. At the end, when the child answer all questions, his/her result will be recorded on the system for the nutrition doctor.

The week average of quiz game will be calculate like below:

$$
\text { qiuz week result }=\frac{\text { total of correct answers }}{\text { total of quizs }}
$$

6. Wrist bond sensor: The wrist bond is built using the SparkFun Triple Axis Accelerometer Breakout - ADXL335 . The ADXL335 is a triple axis MEMS accelerometer with new features including (1) extremely low noise and (2) power consumption of only $320 \mathrm{uA}$. Moreover, it has a full sensing range of $+/-3 \mathrm{~g}$. It is programmed using Arduino and $\mathrm{C}++$. It comes in various colors. It sends data to the cloud server remotely.

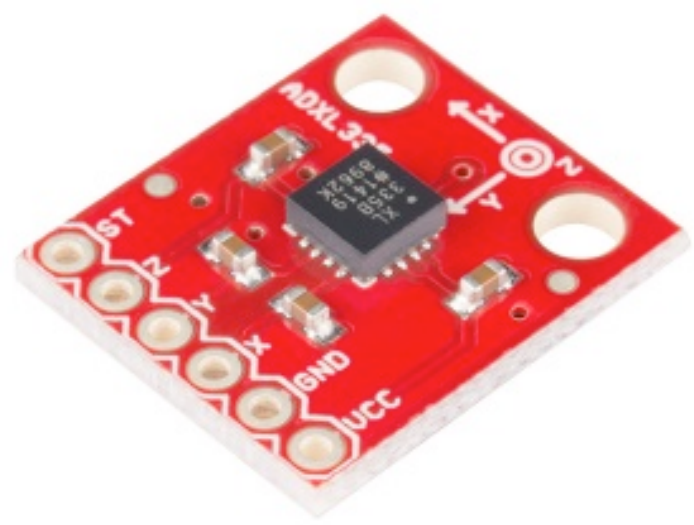

Fig. 8. SparkFun Triple Axis Accelerometer Breakout

However, the system is designed to fit the clinical staff recommendations to improve the obesity management and awareness among obese children and their parent. The developed system including social robot and mobile application is a try to deliver innovation strategy to encourage and enhance obese/overweight children to reduce 
their weight and being more active and more aware of the condition complication. Developer tries to use quiz game to motivate child to involve in educational obesity program in modern way.

\section{Conclusion \& Future works}

The social robotic for obesity management and awareness among children in Saudi Arabia , based on state-of-the-art technological infrastructures, is presented to achieve intensive motivation for obese children to monitor their daily activities and calories consumption, optimized treatment and an ordinary lifestyle for the obese/overweight children in KSA without limits on daily life. Moreover, the obesity educational system designed on the social robot delivers the child with the most important information about obesity management which aim to improve their obesity knowledge, and most attractive and playful for obese children to be more adhere and aware of disease. Future research aims to evaluate the system by running clinical trial study in Saudi Arabia following the regulation of national ethical approval at the university of Tabuk, Saudi Arabia, The trial study will last five months.

This long-term study will assess the efficiency of using the system in improving obesity management and awareness with obese/overweight children in Saudi Arabia. On completion of the clinical trial study, there is need to carry out a suitable testing module and questionnaire to study the system's usability from the clinician staff and obese/overweight children parents and children themselves end. For the evaluation of the system's effectiveness on obese/overweight children, the weight will be checked before and after the trial to allow a comparison between the two readings and also the obesity/health life knowledge test using a questionnaire before and after the study. When the trial is complete, the results will be studied and analyzed using statistical software such as SPSS.

\section{Acknowledgements}

Author gratefully acknowledges the support and assistance of the Club of Robotics, Faculty of Computing and information Technology, University of Tabuk, Saudi Arabia.

\section{References}

[1] Ferrera, L. "Focus on body mass index and health research". New York: Nova Science Publishers. 2006

[2] "Who.int.". (2018). WHO | World Health Organization. [online] Available at: http://www.who.int/home [Accessed 21 May 2018

[3] Worldobesity.org. (2018). World Obesity Federation | Data. [online] Available at: https://www.worldobesity.org/data/ [Accessed 21 May 2018].

[4] Nguyen, N., Nguyen, X., Lane, J. and Wang, P. "Relationship Between Obesity and Diabetes in a US Adult Population: Findings from the National Health and Nutrition Examina- 
Paper-A Social Robotic Obesity Management and Awareness System for Children in Saudi Arabia

tion Survey, 1999-2006”. Obesity Surgery, 21(3), pp.351-355, 2010. https://doi.org/10.1007/s11695-010-0335-4

[5] Eckel, R., Kahn, S., Ferrannini, E., Goldfine, A., Nathan, D., Schwartz, M., Smith, R. and Smith, S. "Obesity and Type 2 Diabetes: What Can Be Unified and What Needs to Be Individualized?". Diabetes Care, 34(6), pp.1424-1430, 2011. https://doi.org/10.2337/dc11$\underline{0447}$

[6] Rössner, S. "Obesity and type 2 diabetes". Practical Diabetes International, 18(8), pp.263264,2001. https://doi.org/10.1002/pdi.261

[7] Al Dhaifallah A, Mwanri L, Aljoudi A.” Childhood obesity in Saudi Arabia: Opportunities and challenges". Saudi J Obesity,p:2-7,2015.

[8] Alotaibi M, "Investigating the Role of Social Robot in improving diabetic Children Management and awareness. S International Journal of Computer Science and Network Security, VOL.17 No.9, 2018.

[9] Al-Taee, M., Kapoor, R., Garrett, C. and Choudhary, P. Acceptability of Robot Assistant in Management of Type 1 Diabetes in Children. Diabetes Technology \& Therapeutics, 18(9), pp.551-554,2016. https://doi.org/10.1089/dia.2015.0428

[10] Saudi Arabia: number of Twitter users 2016 | Statistic [Internet]. Statista. [cited 2018 May 18]. Available from: https://www.statista.com/statistics/558404/number-of-twitter-usersin-saudi-arabia/

\section{$7 \quad$ Author}

Dr. Mohammed Alotaibi received the B.S. in computer Science from King Saud University in 2008 and M.S. degrees in Computer and Information Networks, Essex University, UK (2011)., respectively He got Ph.D. in biomedical informatics from Kingston university London in March 2015. He has served as vice dean for quality and development at scientific research Deanship at University of Tabuk since October 2015 until Feb. 2018. He is now serving as project manager of the university institutional accreditation project and also vice dean for the institute of research and consultancy at the University of Tabuk since May 2018.

\section{Conflict of interest}

The author certify that he has NO affiliations with or involvement in any organization or entity with any financial interest (such as honoraria; educational grants; participation in speakers' bureaus; membership, employment, consultancies, stock ownership, or other equity interest; and expert testimony or patent-licensing arrangements), or non-financial interest (such as personal or professional relationships, affiliations, knowledge or beliefs) in the subject matter or materials discussed in this manuscript.

Article submitted 10 June 2018. Resubmitted 19 July 2018. Final acceptance 22 July 2018. Final version published as submitted by the author. 